\title{
Dynamical realization of magnetic states in a strongly interacting Bose mixture
}

\author{
R. E. Barfknecht, ${ }^{1,2, *}$ A. Foerster,,$^{1, \dagger}$ and N. T. Zinner ${ }^{2, \ddagger}$ \\ ${ }^{1}$ Instituto de Física da UFRGS, Avenida Bento Gonçalves 9500, Porto Alegre, Rio Grande do Sul, Brazil \\ ${ }^{2}$ Department of Physics and Astronomy, Aarhus University, Ny Munkegade 120, Denmark
}

(Received 12 December 2016; published 14 February 2017; corrected 1 May 2017)

\begin{abstract}
We describe the dynamical preparation of magnetic states in a strongly interacting two-component Bose gas in a harmonic trap. By mapping this system to an effective spin-chain model, we obtain the dynamical spin densities and the fidelities for a few-body system. We show that the spatial profiles transit between ferromagnetic and antiferromagnetic states as the intraspecies interaction parameter is slowly increased.
\end{abstract}

DOI: 10.1103/PhysRevA.95.023612

\section{INTRODUCTION}

The recent progress in magneto-optical trapping of ultracold atoms [1] has opened up a new area of experimental development in physics, allowing for the construction of paradigmatic models of quantum mechanics. One of the most important products of these advances is the realization of effective one-dimensional (1D) atomic systems [2-5] where interactions can be tuned via Feshbach [6] or confinementinduced resonances [7,8]. Especially relevant among these 1D experiments is the strongly repulsive bosonic system known as the Tonks-Girardeau gas $[9,10]$. The refinements in manipulation and controlling of cold atoms also enabled the probing of fundamental properties of quantum systems through the construction of few-body ensembles [11-13].

From a theoretical standpoint, the problem of few particles interacting in a harmonic trap has been addressed through different approaches, both exact and approximative [14-20]. The case of strongly interacting atoms, in particular, has been shown to be analogous to an effective 1D spin chain [21-24]. Moreover, strongly interacting few-body systems are suitable for studying the origins of quantum magnetism [25-27] even in models without underlying lattices. Recently, it has been shown that different magnetic orderings can also be induced by adding $p$-wave interactions to the system [28]. While many of these works deal with static properties, the studies involving dynamical features such as spin transport [29], state transfer [30,31], and time evolution following a sudden quench [32] are less numerous. Nonetheless, they are of great experimental interest [29], especially due to their possible applications in spintronics, quantum information processing, and communication [33]. Therefore, a more detailed investigation of quantum dynamics and magnetism in this few-body strongly interacting context is welcome and constitutes the main focus of this work.

Given the motivations above and viewing the possibility of experiments with ultracold few-body Bose mixtures, we consider a model of strongly interacting two-component bosonic atoms in a harmonic trap. It is known that different magnetic states arise as the interactions between bosonic or fermionic atoms are manipulated [27,34]. Here, we specifically

\footnotetext{
*rafael.barfknecht@ufrgs.br

†angela@if.ufrgs.br

${ }^{\ddagger}$ zinner@phys.au.dk
}

show that the spin densities of the system transit between states with clear ferromagnetic (FM) and antiferromagnetic (AFM) profiles as the intraspecies interaction is increased in time. This transition is visible not only in the squared fidelities, but also in the spatial distributions of the spins in the trap.

The paper is organized as follows: In Sec. II we present the Hamiltonian for the strongly interacting two-component bosonic system and the mapping to an effective spin-chain model. By considering the solution of the system in the infinite repulsion limit, we calculate the ground-state spatial densities and the trap-dependent geometric coefficients. The system is then considered to be completely described only by the solution of the spin-chain Hamiltonian. We choose to initialize the system in an eigenstate where the intraspecies interaction is smaller than the interspecies interaction. In Sec. III we proceed to obtain the dynamics of the system: by changing the intraspecies interaction in time and solving the eigenvalue problem at each time step, we can obtain the time evolution of the spin densities. We show that, for increasing intraspecies repulsion, the system evolves from an initial FM state and asymptotically reaches an AFM profile. We demonstrate this by calculating the time evolution of spin densities for different imbalanced systems. In the balanced case, although the spin densities provide less information when compared to the imbalanced situation, the squared fidelities still show the transitions between FM and AFM states. In Sec. IV we present the conclusions and future work perspectives.

\section{HAMILTONIAN AND MAPPING TO AN EFFECTIVE SPIN CHAIN}

We consider a trapped 1D Bose gas with contact interactions and two different bosonic species $(\uparrow, \downarrow)$. The total number of particles is $N=N_{\uparrow}+N_{\downarrow}$, where $N_{\uparrow}$ and $N_{\downarrow}$ are the numbers of particles of species $\uparrow$ and $\downarrow$, respectively. The $N$-body Hamiltonian is given by

$$
\begin{aligned}
H= & \sum_{i}^{N} H_{0}\left(x_{i}\right)+g \sum_{\uparrow \downarrow} \delta\left(x_{i}-x_{j}\right) \\
& +\kappa g \sum_{\uparrow \uparrow} \delta\left(x_{i}-x_{j}\right)+\kappa g \sum_{\downarrow \downarrow} \delta\left(x_{i}-x_{j}\right),
\end{aligned}
$$

where we assume $\hbar=m=1$ and

$$
H_{0}(x)=-\frac{1}{2} \frac{\partial^{2}}{\partial x^{2}}+V(x)
$$




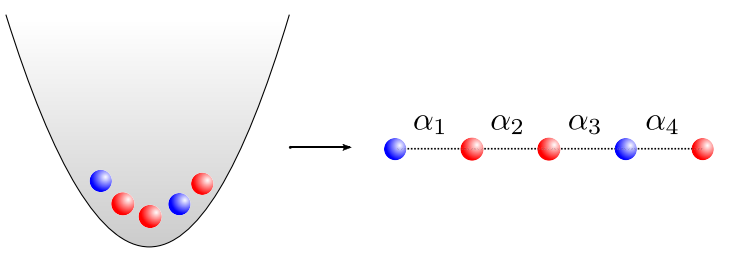

FIG. 1. A system of strongly interacting atoms in a trapping potential can be mapped to an effective spin-chain model where the coefficients $\alpha$ are determined by the geometry of the trap.

is the single-particle Hamiltonian for a given potential $V(x)$ (for harmonic trapping, we have $V(x)=x^{2} / 2$ ). The remaining terms of the Hamiltonian account for the contact interactions between particles of different species (with strength parameter $g$ ) and of the same species (with strength parameter $\kappa g$ ). We consider the length, time, and energy units to be $l=$ $\sqrt{\hbar / m \omega}, \tau=1 / \hbar \omega$, and $E=\hbar \omega$, respectively, where $\omega$ is the longitudinal harmonic confinement frequency [7].

In the limit of infinite repulsion $(1 / g=0)$, the solution of this system is given by the Bose-Fermi mapping [35]. The resulting wave function of hard-core bosons is a symmetrized Slater determinant constructed from the individual eigenstates of the single-particle Hamiltonian (2). Its energy $E_{0}$ is simply the sum of the energies of the lowest occupied energy levels of the potential $V(x)$.

In the limit of strong interactions $(g \gg 1)$, the Hamiltonian (1) can be mapped, up to linear order in $1 / g$, to a spin-chain model given by

$$
H_{s}=E_{0}-\sum_{i=1}^{N-1} \frac{\alpha_{i}}{g}\left[\frac{1}{2}\left(1-\sigma^{i} \cdot \sigma^{i+1}\right)+\frac{1}{\kappa}\left(1+\sigma_{z}^{i} \sigma_{z}^{i+1}\right)\right],
$$

where $\sigma^{i}=\left(\sigma_{x}^{i}, \sigma_{y}^{i}, \sigma_{z}^{i}\right)$ are the Pauli matrices acting on site $i$ and $E_{0}$ is the energy of the hard-core boson (or spinless fermion) system. In the limit of $\kappa \rightarrow \infty$ and positive $g$, the bosons that belong to the same species behave as hardcore particle with respect to each other, while for $\kappa \rightarrow 1$, the interaction strength between all bosons is the same. In the particular case of $\kappa=2$ we have an effective $X X$ model, as summarized in Ref. [22]. The spin model for bosons described in Ref. [24] can be obtained from Eq. (3) by performing a unitary transformation (see Supplemental Material of Ref. [36]).

The coefficients $\alpha$ depend only on the geometry of the trap and are obtained from [22]

$$
\alpha_{i}=\frac{\int_{x_{1}<x_{2}<\cdots<x_{N}-1} d x_{1} \cdots d x_{N-1}\left|\frac{\partial \Phi_{0}^{2}}{\partial x_{N}^{2}}\right|_{x_{N}=x_{i}}^{2}}{\int_{x_{1}<x_{2}<\cdots<x_{N}-1} d x_{1} \cdots d x_{N}\left|\Phi_{0}^{2}\right|},
$$

where $\Phi_{0}\left(x_{1}, \ldots, x_{N}\right)$ is the wave function for spinless fermions. An efficient computational scheme for obtaining the $\alpha$ 's as the number of atoms, $N$, is increased is presented in Ref. [37].

In Fig. 1 we represent the mapping from a strongly interacting 1D system in a harmonic trap to a spin chain characterized by the geometric coefficients $\alpha$. We mainly focus on the $N=5$ problem, for which we obtain $\alpha_{1}=\alpha_{4}=$ 2.16612 and $\alpha_{2}=\alpha_{3}=3.17738$ (since the trap is symmetric,

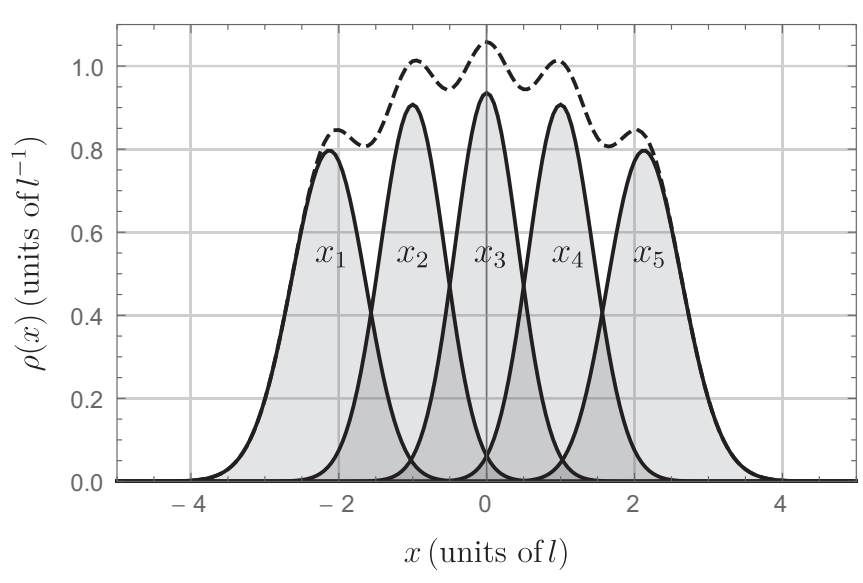

FIG. 2. One-body densities for $N=5$, calculated for the sector $x_{1} \leqslant \cdots \leqslant x_{i} \leqslant \cdots \leqslant x_{5}$. The total density (black dashed curve) is normalized to $N$.

we have $\alpha_{i}=\alpha_{N-i}$ ). Due to a factor of $1 / 2$ in the spin-chain Hamiltonian, our geometric coefficients $\alpha_{i}$ are twice as large as the ones calculated in Ref. [27].

\section{A. One-body correlations for the hard-core boson system}

We focus initially on obtaining the one-body densities for the hard-core boson system, since this accounts for the spatial part of the wave functions. The spatially ordered one-body correlations are given by

$$
\rho^{i}(x)=\int d x_{1} \cdots d x_{N} \delta\left(x_{i}-x\right)\left|\Phi_{0}\left(x_{1}, \ldots, x_{i}, \ldots, x_{N}\right)\right|^{2},
$$

where $\delta(x)$ is the Dirac delta function, and the integration is restricted to the sector $x_{1} \leqslant \cdots \leqslant x_{i} \leqslant \cdots \leqslant x_{N}$. In Fig. 2 we show the densities for the cases of $N=5$. For larger $N$, these integrals become harder to calculate; however, the densities at $x>0$ can be obtained by mirroring the results for $x<0$ [38].

\section{B. Spin densities and initial state for $N=5$}

By taking Eq. (5) for the case of $N=5$, we can calculate the spin densities for the imbalanced cases of three bosons of species $\uparrow$ and two bosons of species $\downarrow\left(N_{\uparrow}=3, N_{\downarrow}=2\right)$ and of four bosons of species $\uparrow$ and one boson of species $\downarrow\left(N_{\uparrow}=4, N_{\downarrow}=1\right)$. To write the separate densities for components $\uparrow$ and $\downarrow$ we must combine the spatial and spinorial contributions; the density for component $\uparrow$, for instance, is given by [25]

$$
\rho_{\uparrow}(x)=\sum_{i=1}^{N} \rho_{\uparrow}^{i}(x),
$$

where $\rho_{\uparrow}^{i}=m_{\uparrow}^{i} \rho^{i}(x)$ and $m_{\uparrow}^{i}$ is the probability of finding a boson of species $\uparrow$ at site $i$ and $\rho^{i}(x)$ is given by Eq. (5). The value of $m^{i}$ for an eigenstate is found by exact diagonalization of Hamiltonian (3), where we consider $g=100$. Since the total spin projection has to be conserved, we choose the basis to be composed only by the desired states, such as 

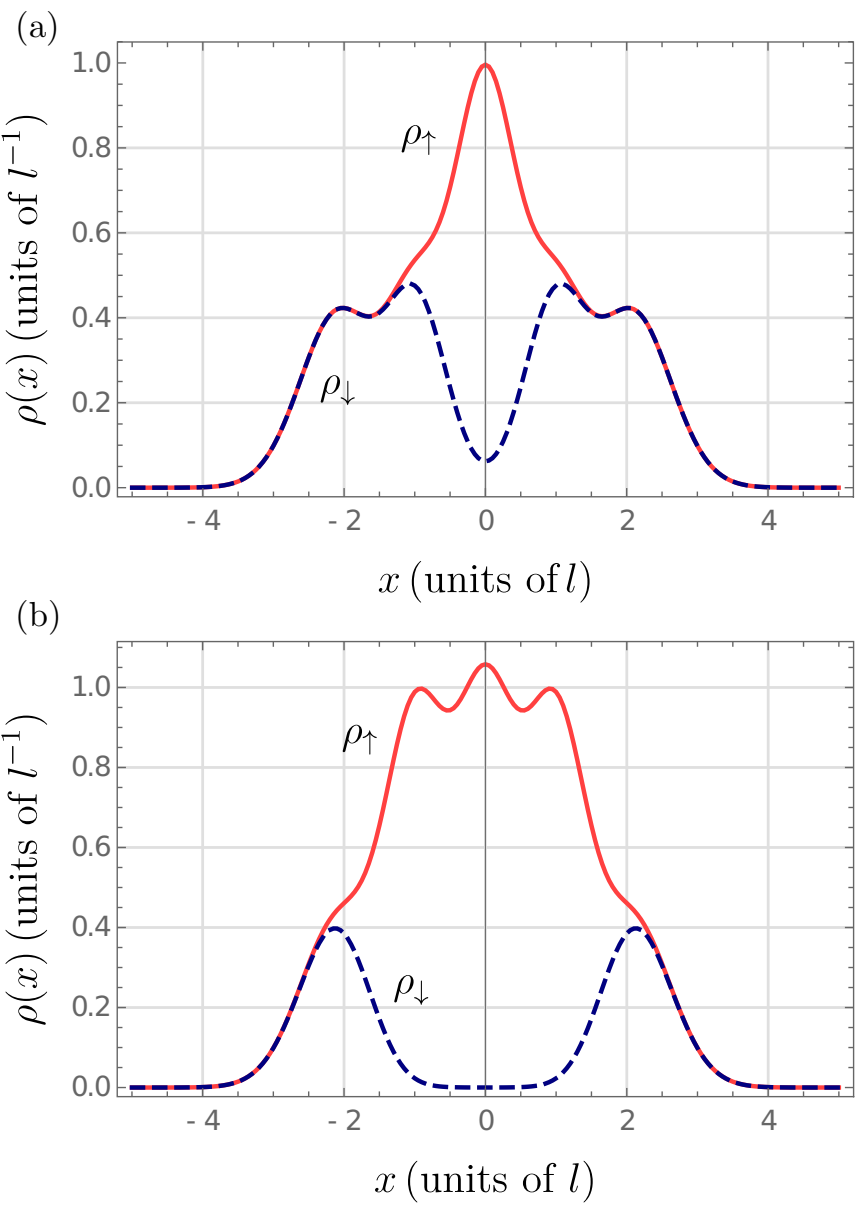

FIG. 3. Spin densities for the initial states, with $\kappa=0.1$, for the (a) $N_{\uparrow}=3, N_{\downarrow}=2$ and (b) $N_{\uparrow}=4, N_{\downarrow}=1$ cases. Solid (light red) and dashed (dark blue) curves describe the spin densities for the $\uparrow$ and $\downarrow$ components, respectively. The separation of different components in the trap indicates a FM behavior.

$|\uparrow \uparrow \uparrow \uparrow \downarrow \downarrow\rangle, \ldots,|\downarrow \downarrow \uparrow \uparrow \uparrow \uparrow\rangle$ for the $N_{\uparrow}=3, N_{\downarrow}=2$ case and $|\uparrow \uparrow \uparrow \uparrow \uparrow \downarrow\rangle, \ldots,|\downarrow \uparrow \uparrow \uparrow \uparrow \uparrow\rangle$ for the $N_{\uparrow}=4, N_{\downarrow}=1$ case.

The complete ground-state wave function, including the spatial and spin eigenfunctions, must take into account the combined symmetry of these states. For instance, for a bosonic system, the ground state of the spin Hamiltonian is symmetric, which means that the spatial part of the wave function must also be symmetric to account for a totally symmetric state [38]. In the following sections, however, we do not take the complete wave function into account since we are dealing directly with the spin densities given by Eq. (6).

We now construct the initial states of the system by choosing the ground states in which the intraspecies interaction is smaller than the interspecies interaction $(\kappa=0.1)$. In Fig. 3 we show the spin densities for the imbalanced cases of $N_{\uparrow}=3, N_{\downarrow}=2$ and $N_{\uparrow}=4, N_{\downarrow}=1$. At this point, due to the difference in the interaction strengths, the species tend to separate in the trap. The densities profiles for $\kappa<1$ show a ferromagnetic order [19] of the Ising type, as opposed to the case where $\kappa=1$, which is addressed next. In Fig. 3(b), we see a density that is similar to that of the Bose polaron [26], where a strongly interacting impurity is pushed to the edges of the system.

\section{DYNAMICAL PREPARATION OF MAGNETIC STATES}

\section{A. Imbalanced system}

We now consider the time evolution of the system for a slow increase in the intraspecies interaction parameter $\kappa$. We take $\kappa$ varying in the interval $[0.1,10.1]$. The eigenfunctions of the spin-chain Hamiltonian thus evolve as

$$
\left|\chi\left(t_{f}\right)\right\rangle=U\left(t_{f}, t_{0}\right)\left|\chi_{0}\right\rangle,
$$

where $U\left(t_{f}, t_{0}\right)$ is the time-evolution operator and $\left|\chi_{0}\right\rangle$ is the initial state. Since the Hamiltonian is time dependent, we can break the time evolution in several steps,

$$
\left|\chi\left(t_{f}\right)\right\rangle=U\left(t_{N}, t_{N-1}\right) \cdots U\left(t_{2}, t_{1}\right) U\left(t_{1}, t_{0}\right)\left|\chi_{0}\right\rangle,
$$

increasing $\Delta \kappa=1 \times 10^{-5}$ and taking the Hamiltonian to be constant at each time step.

During the first steps of the time evolution $(\kappa \sim 0.1)$, the change in energy at each step is larger than the spin gap $\Delta E$ between the ground state and the first excited state of

(a)

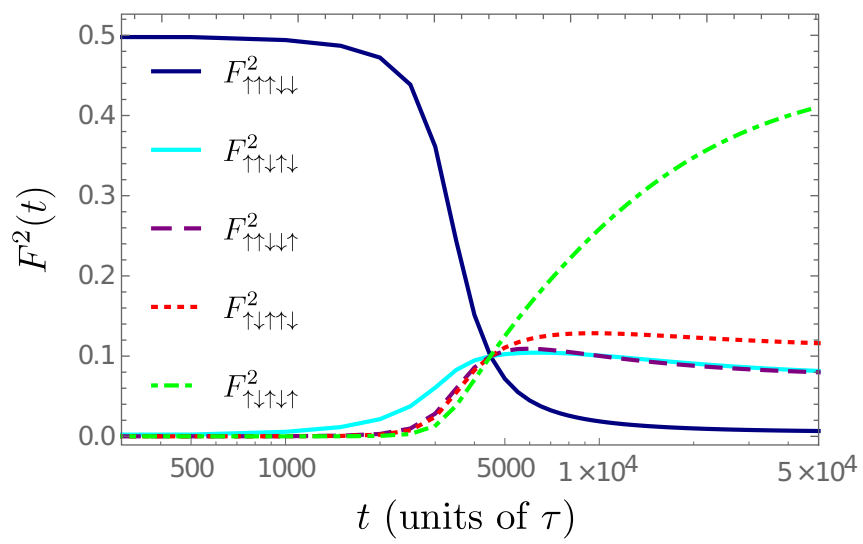

(b)

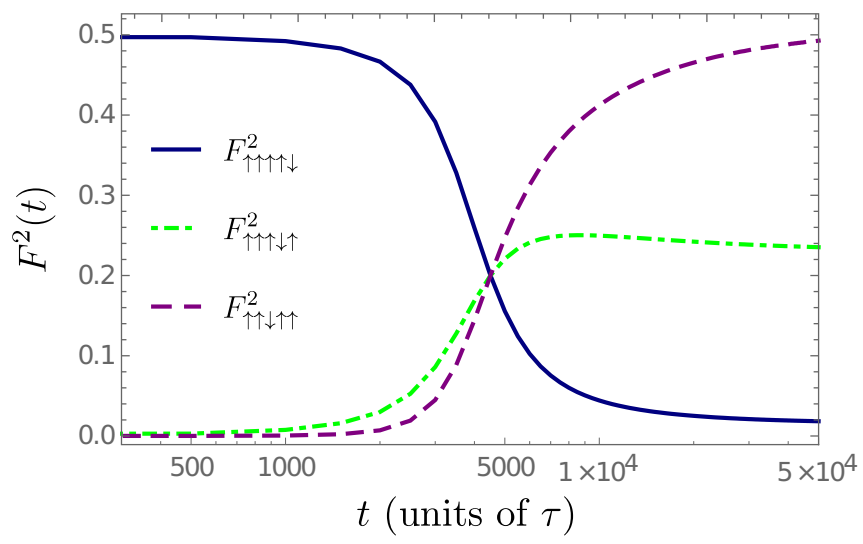

FIG. 4. Time evolution of the squared fidelities for (a) the $N_{\uparrow}=3, N_{\downarrow}=2$ and (b) the $N_{\uparrow}=4, N_{\downarrow}=1$ cases. Identical results for symmetric states (e.g., $F_{\downarrow \downarrow \uparrow \uparrow \uparrow}^{2}=F_{\uparrow \uparrow \uparrow \downarrow \downarrow}^{2}$ ) are omitted. At $t=$ $0.45 \times 10^{4}[\tau]$, the system reaches the Heisenberg-type FM state characterized by $\kappa=1$, where the values for all the projections are the same. 
(a)

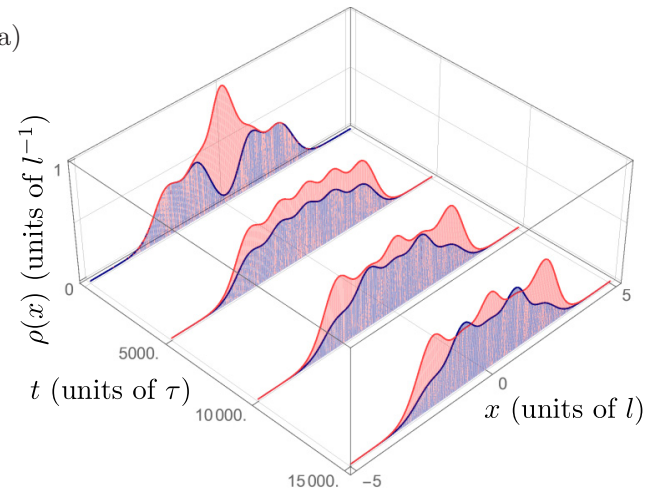

(b)

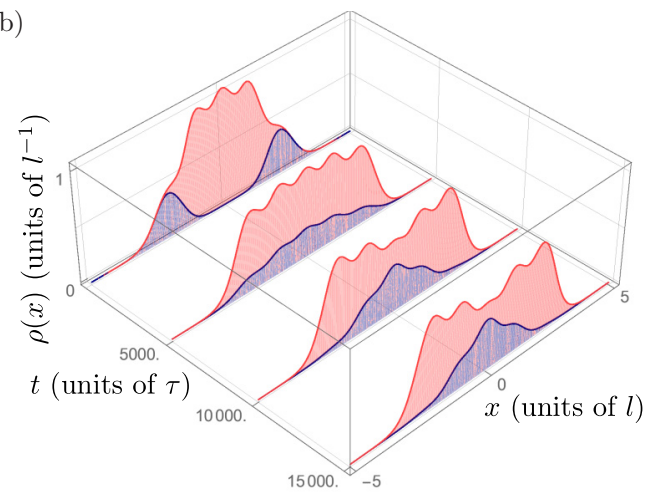

FIG. 5. Initial part of the time evolution $\left(t \leqslant 1.5 \times 10^{4}[\tau]\right)$ of the spin densities for the cases of (a) $N_{\uparrow}=3, N_{\downarrow}=2$ and (b) $N_{\uparrow}=4$, $N_{\downarrow}=1$. Light (red) and dark (blue) curves indicate the $\uparrow$ and $\downarrow$ components, respectively. Initial profiles (up to $t=0.5 \times 10^{4}[\tau]$ ) indicate FM states. At around $t=10^{4}[\tau]$, AFM profiles start to arise.
Hamiltonian (3). This means that, initially, the evolution of the system is not adiabatic. Therefore, the whole set of eigenvalues and eigenstates of the spin chain must be calculated for all times. The energy gap between the ground state and the first excited state of the spatial wave function, however, is given by $\hbar \omega \gg \Delta \kappa \hbar \omega$, so we can neglect the excited states of $\Phi\left(x_{1}, x_{2}, \ldots, x_{N}\right)$.

The recursion formula for the time evolution of the spinwave function is then given by

$$
\left|\chi_{i+1}\right\rangle=\sum_{i=1}^{v} c_{n}^{i+1} e^{-i E_{n}^{i+1} \Delta t}\left|\phi_{n}^{i+1}\right\rangle,
$$

where $i$ denotes the time step, $E_{n}^{i+1}$ and $\phi_{n}^{i+1}$ are the eigenvalues and eigenvectors of the Hamiltonian (3) at step $i+1, c_{n}^{i+1}=\left\langle\phi_{n}^{i+1} \mid \chi_{i}\right\rangle$, and $v$ is the number of eigenstates (the total time evolution may be thought of as a succession of small quenches, with fixed $\Delta t=0.05[\tau])$. In Fig. 4 we show the dynamical squared fidelities $F_{\xi}^{2}(t)$, with $F_{\xi}(t)=|\langle\xi \mid \chi(t)\rangle|$, where $|\xi\rangle$ is some basis state (e.g., $|\xi\rangle=|\uparrow \uparrow \uparrow \downarrow \downarrow\rangle$ for the $N_{\uparrow}=3, N_{\downarrow}=2$ case). Since the eigenstates are composed of linear combinations of the symmetric basis states, in Fig. 4, the results of the squared fidelities for states such as $|\uparrow \uparrow \uparrow \downarrow \downarrow\rangle$ and $|\downarrow \downarrow \uparrow \uparrow \uparrow\rangle$ are identical. Therefore, we choose to omit the results for the symmetric cases.

In Fig. 5 we present the time evolution (up to $t=1.5 \times$ $\left.10^{4}[\tau]\right)$ of the spin densities for the two imbalanced cases under consideration. We see that, for $0<\kappa \leqslant 1$, the system evolves through a FM phase. This phase is characterized first by the separation of the two components in the trap and then (around $t=0.45 \times 10^{4}[\tau]$ and $\kappa \sim 1$ ) by the typical densities of two-component bosonic systems with strong repulsive

(b)

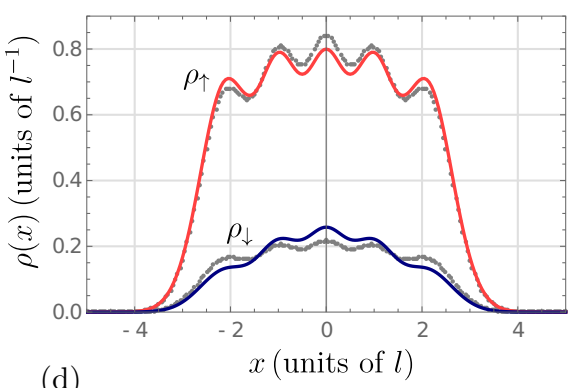

(c)

(d)

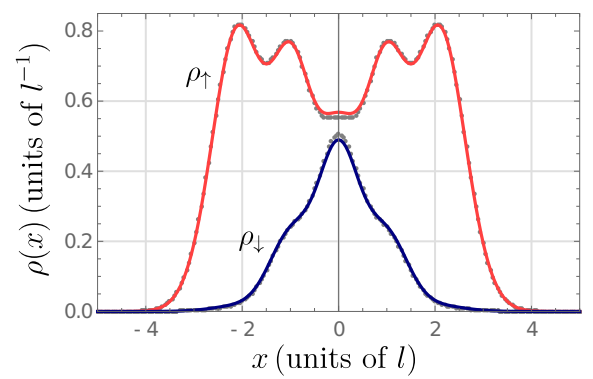

FIG. 6. Intermediate and final profiles for the time evolution of spin densities. Light (red) and dark (blue) curves indicate the $\uparrow$ and $\downarrow$ components, respectively. Upper panels show the profiles at $\kappa=1.1\left(t=0.5 \times 10^{4}[\tau]\right)$ for the (a) $N_{\uparrow}=3, N_{\downarrow}=2$ and (b) $N_{\uparrow}=4, N_{\downarrow}=1$ cases. The gray dots correspond to the results obtained with $g=-100$ and $\kappa \rightarrow \infty$. The lower panels show the final profiles $\left(t=0.5 \times 10^{5}[\tau]\right.$, $\kappa \sim 10$ ) for the (c) $N_{\uparrow}=3, N_{\downarrow}=2$ and (d) $N_{\uparrow}=4, N_{\downarrow}=1$ cases, now compared to the limiting case of $g=100$ and $\kappa \rightarrow \infty$ (gray dots). 
interactions [39]. For the particular case of $\kappa=1$, all the interactions between bosons are identical. The magnetic order is of the Heisenberg type with isotropic interactions, and the squared fidelities assume the same values for all basis states, as we can observe in Fig. 4. In this regime, the densities show the profiles that characterize itinerant ferromagnetism (notice the distinction between the profiles in this regime and in the Ising-type FM regime of $\kappa<1$ ). In Figs. 6(a) and 6(b), we show the comparison between the slice at $t=0.5 \times 10^{4}[\tau]$ (which corresponds to $\kappa=1.1$ ) and the results obtained by exact diagonalization of Hamiltonian (3) with $g=-100$ and $\kappa \rightarrow \infty$. In this limit, the densities reproduce the results expected for the strongly attractive two-component fermionic gas [27].

As the intraspecies interaction becomes stronger $(\kappa>1)$, an AFM profile starts to arise. This is translated in Fig. 4 as the increase of the projections over the states $|\uparrow \downarrow \uparrow \downarrow \uparrow\rangle$ [green dash-dotted curve in Fig. 4(a)] and $|\uparrow \uparrow \downarrow \uparrow \uparrow\rangle$ [purple dashed curve in Fig. 4(b)]. This effect can be seen already during the first part of the time evolution $\left(t \geqslant 1.0 \times 10^{4}[\tau]\right)$, as it is shown in Fig. 5. Finally, for $\kappa \gg 1$, the AFM profiles become more pronounced (rigorously, an AFM state can only be reached for $\kappa \rightarrow \infty$ ). In Figs. 6(c) and 6(d), we compare the final densities at $\kappa=10.1$ to the results obtained for $\kappa \rightarrow \infty$. The results in this case match the AFM states of strongly repulsive two-component fermions. It is important to point out that while the spin densities may reproduce results of fermionic systems in certain limits, this may not be true for other correlations (e.g., the momentum distribution).

The total time evolution is given by $t_{f}=0.5 \times 10^{5}[\tau]$. In current experimental setups, the inverse frequency $\tau$ is of the order of $100 \mu \mathrm{s}$ [13]. This results in a total time of $5 \mathrm{~s}$ for the process we are considering, which is a relatively long time for experiments with ultracold atoms. We point out, however, that the transition from FM to AFM-like profiles is manifested early on in this time evolution. This means that these effects could conceivably be observed in smaller time intervals. Alternatively, increasing the trap frequency could result in a smaller time scale for the process, where the intraspecies interaction could be tuned from weak to strong in a shorter time.

\section{B. Balanced system}

We consider now a balanced system composed of $N_{\uparrow}=2$, $N_{\downarrow}=2$. Once again we choose an initial state where the intraspecies interaction is smaller than the interspecies interaction $(\kappa=0.1)$.

In Fig. 7(a), although a FM profile is still observed, there is no visible separation of components in the trap, due to the fact that the system is now balanced. Since the probabilities of finding spin-up and -down bosons at each site are always the same, the spin densities do not change in time as $\kappa$ increases. However, the squared fidelities display a behavior analogous to that of the imbalanced cases, where the AFM states become dominant as $\kappa \rightarrow \infty$. Unlike the imbalanced cases, the AFM state for $N_{\uparrow}=2, N_{\downarrow}=2$ is composed by the linear combination of $|\uparrow \downarrow \uparrow \downarrow\rangle$ and $|\downarrow \uparrow \downarrow \uparrow\rangle$.
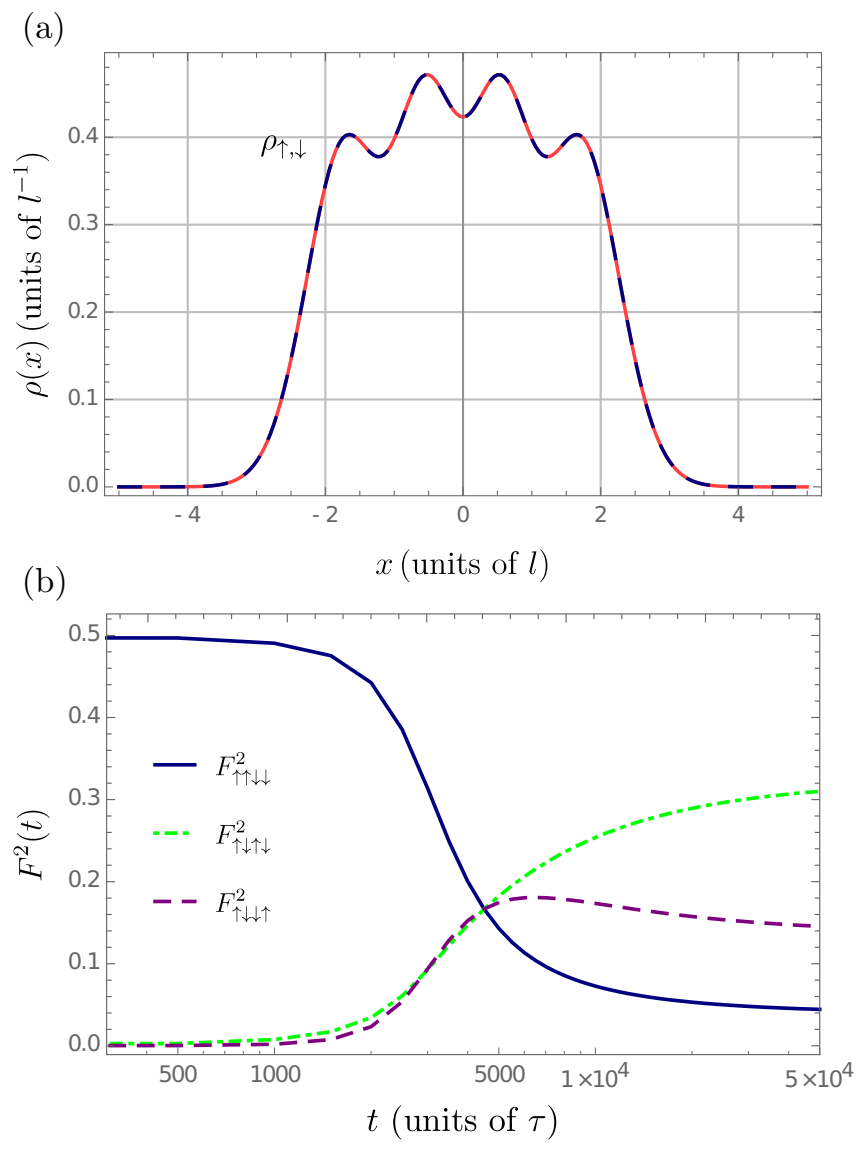

FIG. 7. (a) Spin densities for a balanced $N_{\uparrow}=2, N_{\downarrow}=2$ system. Solid (light red) and dashed (dark blue) curves indicate the $\uparrow$ and $\downarrow$ components, respectively. Due to the absence of imbalance, there is no change in the spin densities as $\kappa$ is varied. (b) The squared fidelities display a transition from FM to AFM states similar to those observed in the imbalanced cases.

\section{CONCLUSIONS}

We showed that different magnetic states can be achieved by dynamically changing the intraspecies interactions of a two-component strongly repulsive few-body bosonic gas. Due to the strong interactions, this model can be mapped to an effective spin chain with solutions that completely determine the state of the system. By slowly increasing the interactions between the identical bosons, we are able to keep the spatial densities fixed in the ground state, while the spin eigenstates evolve in time. The spin densities then display a clear transition between FM and AFM profiles. In addition, during this evolution the system exhibits results that match the limiting cases of strong interspecies attraction or repulsion, depending only on the tuning of the parameter $\kappa$.

Future elaborations of the work presented here could be based on the study of quench dynamics in strongly interacting bosonic mixtures, now taking into account the excited states of the spatial wave function. Other interesting extensions would include the dynamics of larger ensembles of interacting bosonic gases, which could help bridge the gap between the few-body and many-body landscapes. 


\section{ACKNOWLEDGMENTS}

The authors thank Xiaoling Cui and Carlos Kuhn for useful comments. R.E.B. thanks Marcos Pérez for inspiring discussions. The following agencies-Conselho Nacional de De- senvolvimento Científico e Tecnológico (CNPq), the Danish Council for Independent Research DFF Natural Sciences, and the DFF Sapere Aude program—are gratefully acknowledged for financial support.
[1] I. Bloch, Ultracold quantum gases in optical lattices, Nat. Phys 1, 23 (2005).

[2] H. Moritz, T. Stöferle, M. Köhl, and T. Esslinger, Exciting Collective Oscillations in a Trapped 1D Gas, Phys. Rev. Lett. 91, 250402 (2003).

[3] T. Kinoshita, T. Wenger, and D. S. Weiss, A quantum Newton's cradle, Nature (London) 440, 900 (2006).

[4] E. Haller, M. Gustavsson, M. J. Mark, J. G. Danzl, R. Hart, G. Pupillo, and H.-C. Nägerl, Realization of an excited, strongly correlated quantum gas phase, Science 325, 1224 (2009).

[5] E. Haller, R. Hart, M. J. Mark, J. G. Danzl, L. Reichsollner, M. Gustavsson, M. Dalmonte, G. Pupillo, and H.-C. Nagerl, Pinning quantum phase transition for a Luttinger liquid of strongly interacting bosons, Nature (London) 466, 597 (2010).

[6] C. Pethick and H. Smith, Bose-Einstein Condensation in Dilute Gases (Cambridge University Press, Cambridge, U.K., 2002).

[7] M. Olshanii, Atomic Scattering in the Presence of an External Confinement and a Gas of Impenetrable Bosons, Phys. Rev. Lett. 81, 938 (1998).

[8] E. Haller, M. J. Mark, R. Hart, J. G. Danzl, L. Reichsöllner, V. Melezhik, P. Schmelcher, and H.-C. Nägerl, ConfinementInduced Resonances in Low-Dimensional Quantum Systems, Phys. Rev. Lett. 104, 153203 (2010).

[9] B. Paredes, A. Widera, V. Murg, O. Mandel, S. Folling, I. Cirac, G. V. Shlyapnikov, T. W. Hansch, and I. Bloch, Tonks-Girardeau gas of ultracold atoms in an optical lattice, Nature (London) 429, 277 (2004).

[10] T. Kinoshita, T. Wenger, and D. S. Weiss, Observation of a onedimensional Tonks-Girardeau gas, Science 305, 1125 (2004).

[11] G. Zürn, F. Serwane, T. Lompe, A. N. Wenz, M. G. Ries, J. E. Bohn, and S. Jochim, Fermionization of Two Distinguishable Fermions, Phys. Rev. Lett. 108, 075303 (2012).

[12] A. N. Wenz, G. Zürn, S. Murmann, I. Brouzos, T. Lompe, and S. Jochim, From few to many: Observing the formation of a Fermi sea one atom at a time, Science 342, 457 (2013).

[13] S. Murmann, F. Deuretzbacher, G. Zürn, J. Bjerlin, S. M. Reimann, L. Santos, T. Lompe, and S. Jochim, Antiferromagnetic Heisenberg Spin Chain of a Few Cold Atoms in a One-Dimensional Trap, Phys. Rev. Lett. 115, 215301 (2015).

[14] I. Brouzos and P. Schmelcher, Construction of Analytical ManyBody Wave Functions for Correlated Bosons in a Harmonic Trap, Phys. Rev. Lett. 108, 045301 (2012).

[15] D. Rubeni, A. Foerster, and I. Roditi, Two interacting fermions in a one-dimensional harmonic trap: Matching the Bethe ansatz and variational approaches, Phys. Rev. A 86, 043619 (2012).

[16] B. Wilson, A. Foerster, C. Kuhn, I. Roditi, and D. Rubeni, A geometric wave function for a few interacting bosons in a harmonic trap, Phys. Lett. A 378, 1065 (2014).
[17] A. G. Volosniev, D. V. Fedorov, A. S. Jensen, M. Valiente, and N. T. Zinner, Strongly interacting confined quantum systems in one dimension, Nat. Commun. 5, 5300 (2014).

[18] M. A. García-March, B. Juliá-Díaz, G. E. Astrakharchik, J. Boronat, and A. Polls, Distinguishability, degeneracy, and correlations in three harmonically trapped bosons in one dimension, Phys. Rev. A 90, 063605 (2014).

[19] A. S. Dehkharghani, A. G. Volosniev, and N. T. Zinner, Quantum impurity in a one-dimensional trapped Bose gas, Phys. Rev. A 92, 031601 (2015).

[20] R. E. Barfknecht, A. S. Dehkharghani, A. Foerster, and N. T. Zinner, Correlation properties of a three-body bosonic mixture in a harmonic trap, J. Phys. B 49, 135301 (2016).

[21] F. Deuretzbacher, K. Fredenhagen, D. Becker, K. Bongs, K. Sengstock, and D. Pfannkuche, Exact Solution of Strongly Interacting Quasi-One-Dimensional Spinor Bose Gases, Phys. Rev. Lett. 100, 160405 (2008).

[22] A. G. Volosniev, D. Petrosyan, M. Valiente, D. V. Fedorov, A. S. Jensen, and N. T. Zinner, Engineering the dynamics of effective spin-chain models for strongly interacting atomic gases, Phys. Rev. A 91, 023620 (2015).

[23] L. Yang, L. Guan, and H. Pu, Strongly interacting quantum gases in one-dimensional traps, Phys. Rev. A 91, 043634 (2015).

[24] L. Yang and X. Cui, Effective spin-chain model for strongly interacting one-dimensional atomic gases with an arbitrary spin, Phys. Rev. A 93, 013617 (2016).

[25] F. Deuretzbacher, D. Becker, J. Bjerlin, S. M. Reimann, and L. Santos, Quantum magnetism without lattices in strongly interacting one-dimensional spinor gases, Phys. Rev. A 90, 013611 (2014).

[26] A. Dehkharghani, A. Volosniev, J. Lindgren, J. Rotureau, C. Forssén, D. Fedorov, A. Jensen, and N. Zinner, Quantum magnetism in strongly interacting one-dimensional spinor Bose systems, Sci. Rep. 5, 10675 (2015).

[27] P. Massignan, J. Levinsen, and M. M. Parish, Magnetism in Strongly Interacting One-Dimensional Quantum Mixtures, Phys. Rev. Lett. 115, 247202 (2015).

[28] L. Yang, X. Guan, and X. Cui, Engineering quantum magnetism in one-dimensional trapped Fermi gases with $p$-wave interactions, Phys. Rev. A 93, 051605 (2016).

[29] T. Fukuhara, A. Kantian, M. Endres, M. Cheneau, P. Schausz, S. Hild, D. Bellem, U. Schollwock, T. Giamarchi, C. Gross, I. Bloch, and S. Kuhr, Quantum dynamics of a mobile spin impurity, Nat. Phys. 9, 235 (2013).

[30] A. G. Volosniev, H.-W. Hammer, and N. T. Zinner, Simulation of time-dependent Heisenberg models in one dimension, Phys. Rev. B 93, 094414 (2016).

[31] N. J. S. Loft, O. V. Marchukov, D. Petrosyan, and N. T. Zinner, Tunable self-assembled spin chains of strongly interacting cold atoms for demonstration of reliable quantum state transfer, New J. Phys. 18, 045011 (2016). 
[32] L. Yang and H. Pu, Bose-Fermi mapping and a multibranch spin-chain model for strongly interacting quantum gases in one dimension: Dynamics and collective excitations, Phys. Rev. A 94, 033614 (2016).

[33] S. Bose, Quantum communication through spin chain dynamics: An introductory overview, Contemp. Phys. 48, 13 (2007).

[34] H. Hu, L. Pan, and S. Chen, Strongly interacting onedimensional quantum gas mixtures with weak $p$-wave interactions, Phys. Rev. A 93, 033636 (2016).

[35] M. Girardeau, Relationship between systems of impenetrable bosons and fermions in one dimension, J. Math. Phys. 1, 516 (1960).
[36] O. V. Marchukov, A. G. Volosniev, M. Valiente, D. Petrosyan, and N. T. Zinner, Quantum spin transistor with a Heisenberg spin chain, Nat. Commun. 7, 13070 (2016).

[37] N. Loft, L. Kristensen, A. Thomsen, A. Volosniev, and N. Zinner, CONAN: The cruncher of local exchange coefficients for strongly interacting confined systems in one dimension, Comput. Phys. Commun. 209, 171 (2016).

[38] F. Deuretzbacher, D. Becker, and L. Santos, Momentum distributions and numerical methods for strongly interacting one-dimensional spinor gases, Phys. Rev. A 94, 023606 (2016).

[39] Y. J. Hao and S. Chen, Ground-state properties of interacting two-component Bose gases in a one-dimensional harmonic trap, Eur. Phys. J. D 51, 261 (2009). 